\title{
Evaluation of Subclinical Left Ventricular Systolic Dysfunction in Chronic Asymptomatic Alcoholics by Speckle Tracking Echocardiography
}

\author{
Murathan Kucuk, ${ }^{1}$ Can Ramazan Oncel, ${ }^{2}$ Aytul Belgi Yıldırım, \\ Fatih Canan, ${ }^{3}$ and Mehmet Murat Kuloglu ${ }^{3}$ \\ ${ }^{1}$ Akdeniz University Medical Faculty, Department of Cardiology, Antalya, Turkey \\ ${ }^{2}$ Ataturk State Hospital, Department of Cardiology, Antalya, Turkey \\ ${ }^{3}$ Akdeniz University Medical Faculty, Department of Psychiatry, Antalya, Turkey \\ Correspondence should be addressed to Can Ramazan Oncel; r_oncel@hotmail.com
}

Received 29 November 2016; Accepted 19 March 2017; Published 29 March 2017

Academic Editor: Hongqun Liu

Copyright (C) 2017 Murathan Kucuk et al. This is an open access article distributed under the Creative Commons Attribution License, which permits unrestricted use, distribution, and reproduction in any medium, provided the original work is properly cited.

\begin{abstract}
By using two-dimensional speckle tracking echocardiography, we aimed to investigate the structural and functional changes on myocardium in chronic asymptomatic alcoholics without any cardiovascular disease. Forty-one consecutive asymptomatic male alcoholics who were admitted to the outpatient alcoholism unit and 30 age matched healthy male volunteers selected as the control group were enrolled in the study. The study group were investigated by using standard two-dimensional echocardiography and speckle tracking echocardiography. The left ventricular (LV) global longitudinal strain and LV global circumferential strain were significantly lower in alcoholics when compared with control subjects. There was no difference in global radial strain between the two groups. To demonstrate the effect of total life time dose of ethanol (TLDE) on echocardiographic abnormalities, we assessed the correlation analysis. There was a nonsignificant weak correlation between global LV circumferential strain and TLDE $(r=0.27$, $p=0.083$ ). Speckle tracking echocardiography derived left ventricular systolic function was impaired in chronic alcoholic patients when compared with healthy controls.
\end{abstract}

\section{Introduction}

Chronic excessive alcohol consumption is related with cardiomyopathy where alcoholic cardiomyopathy has been seen in about $10 \%$ of alcoholics and prevalence ranges from $23 \%$ to $40 \%$ [1].

Transthoracic echocardiography can detect mild left ventricular hypertrophy, diastolic dysfunction, and left ventricular dilatation in asymptomatic alcoholics [2]. Although reduced left ventricular ejection fraction can be documented by conventional echocardiography, it is important to find a method for the detection of subclinical LV systolic dysfunction in these patients. Predicting early cardiac toxicity of chronic alcohol consumption in patients at high risk for developing alcoholic cardiomyopathy will be helpful, since an early withdrawal of alcohol may improve left ventricular systolic function and prognosis in this group.

Our study aims to analyze the structural and functional changes on myocardium using two-dimensional speckle tracking echocardiography in chronic asymptomatic alcoholics without any cardiovascular disease and any disease which could impair myocardial functions.

\section{Methods}

Forty-one consecutive asymptomatic male alcoholics who were admitted to the outpatient alcoholism unit which is located in Mediterranean region of Turkey were enrolled in this study. Thirty age matched healthy male volunteers were 
selected as the control group. None of them had any history, signs, or symptoms of cardiovascular diseases.

The exclusion criteria were a history of congenital heart disease, hypertension, diabetes mellitus, coronary artery disease, valvular heart disease, atrial fibrillation, and systemic and metabolic disease such as chronic liver disease or renal dysfunction. All the patients were questioned about the amount and duration of alcohol consumption.

Total lifetime dose of ethanol (TLDE) was calculated by first multiplying the daily consumption of ethanol by the number of days of the periods of exposure to alcohol and then dividing the product by the body weight of the patient [3].

2.1. Echocardiography. Echocardiographic examinations including conventional, pulsed Doppler, tissue Doppler, and speckle tracking echocardiography were performed in 41 alcoholics and 30 age matched controls. Echocardiographic study was done by ultrasound machine GE-Vingmed vivid 7 system (GE-Vingmed Ultrasound AS, Horten, Norway) and 3S-RS (3.5 mhz) probe.

Speckle tracking echocardiography analysis was performed from apical and parasternal short axis views, respectively. Standard grayscale 2D images were obtained at a frame rate of 70-90 frames/s during three cardiac cycles and software package (Echopac PC, version 6.0, GE healthcare) was used for offline analysis. Endocardial border of the left ventricle was traced manually from the end systolic frame. The software automatically detected the epicardial border and created a region of interest which was adjusted manually to include the entire myocardial wall. Apical and short axis views of left ventricle were processed by software to select natural acoustic markers. Then these markers were tracked frame by frame during cardiac cycle to measure strain and strain rate of left ventricle at any point of the myocardium. To obtain optimal tracking, necessary corrections were done for the quality tracking verification and region of interest modification. All echocardiographic measurements were done according to the guidelines of American Society of Echocardiography.

2.2. Clinical and Laboratory Evaluations. After a complete physical examination, anthropometric measurements of height and weight were done and body mass index (BMI, $\mathrm{kg} / \mathrm{m}^{2}$ ) and body surface area $\left(\mathrm{BSA}, \mathrm{m}^{2}\right.$ ) of all subjects were calculated. Venous blood count was obtained from all subjects on admission as a routine part of clinical examination. A detailed questionnaire was applied to evaluate the real dose of alcohol consumed and then confirmed by family members because of the known characteristics of alcoholics.

2.3. Statistical Analysis. Continuous and categorical variables were expressed as mean \pm standard deviation (SD) and percentages, respectively. The Kolmogorov-Smirnov test was used to verify the normality of the distribution of continuous variables. Continuous variables were compared using oneway ANOVA test. Categorical variables were compared using chi-square test $(\chi 2)$ or Fisher's exact test, as appropriate. Spearman's test was used for correlation analysis. A $p$ value of less than 0.05 was regarded significant for all analyses. Analyses were performed with PASW 18 (SPSS/IBM, Chicago, IL, USA) software and two-tailed $p$ value less than 0.05 was considered statistically significant.

2.4. Inter- and Intraobserver Variability. We reported strain measurements in 20 random alcoholics or control subjects three weeks after the first evaluation for determining intraobserver variability. Also these measurements were done by another observer for determining interobserver variability. The Bland-Altman analysis was calculated for interobserver and intraobserver variability.

\section{Results}

Thirty healthy subjects and 41 alcoholics were evaluated in the study. Detailed demographic, clinical, and biochemical variables of two groups are shown in Table 1 . The $\mathrm{M}$ mode, two-dimensional, pulse Doppler, and tissue Doppler echocardiographic parameters of two groups were given in Table 2.

Left ventricular ejection fraction did not show any difference between healthy subjects and the alcoholics. Compared to controls, alcoholic group had a significant difference in left atrium diameter $(31.83 \pm 0.47,34.13 \pm 0.56, p=0.003)$. There were no differences in LV mass index, EDD, ESD, E/A ratio, IVRT, and IVCT among two groups. Deceleration time was longer in alcoholics $(186.14 \pm 4.49,204 \pm 6.17, p=0.019)$.

Alcoholic patients had consumed a mean daily dose of ethanol of $186.64 \pm 7 \mathrm{~g}$ over a period of $20.38 \pm 1.37$ years with a mean TLDE of $19.94 \pm 12.17 \mathrm{~kg}$ ethanol $/ \mathrm{kg}$ body weight.

3.1. Left Ventricular Deformation Analysis. The LV global longitudinal strain and LV global circumferential strain were significantly lower in alcoholics when compared with control subjects. There was no difference in global radial strain between two groups. Strain measurements of two groups were given in Table 3. To demonstrate the effect of TLDE on echocardiographic abnormalities, we used the correlation analysis. There was a nonsignificant weak correlation between global LV circumferential strain and TLDE $(r=0.27, p=$ 0.083).

Using two-dimensional speckle tracking echocardiography to evaluate alcoholic cardiac damage, inter- and intraobserver variability results showed a good reproducibility and small variability.

\section{Discussion}

In our study we demonstrated that left ventricular global longitudinal strain and left ventricular global circumferential strain were deteriorated in chronic alcoholic patients. It has been shown that global longitudinal strain is a quantitative index for global left ventricle function [4]. Two-dimensional speckle tracking echocardiography allowed us to detect early systolic dysfunction in chronic alcoholic patients with a preserved LVEF.

Chronic alcoholic consumption causes several histological and cellular changes in the myocardium. These changes 
TABLE 1: Demographic, clinical, and biochemical variables of alcoholic patients and controls.

\begin{tabular}{|c|c|c|c|}
\hline & $\begin{array}{c}\text { Alcoholic patients } \\
\qquad N=41\end{array}$ & $\begin{array}{l}\text { Healthy controls } \\
\qquad N=30\end{array}$ & $p$ \\
\hline Age (years) & $43.17 \pm 1.4$ & $38.0 \pm 1.73$ & 0.022 \\
\hline $\mathrm{BMI}\left(\mathrm{kg} / \mathrm{m}^{2}\right)$ & $24.79 \pm 0.68$ & $26.60 \pm 0.82$ & 0.093 \\
\hline Body surface area $\left(\mathrm{m}^{2}\right)$ & $1.89 \pm 0.03$ & $1.96 \pm 0.03$ & 0.123 \\
\hline Systolic blood pressure (mmHg) & $119.29 \pm 1.7$ & $118.77 \pm 1.54$ & 0.829 \\
\hline Diastolic blood pressure (mmHg) & $77.86 \pm 1.0$ & $73.83 \pm 1.21$ & 0.012 \\
\hline AST (U/L) & $65.33 \pm 12.47$ & $22.39 \pm 1.21$ & 0.005 \\
\hline $\operatorname{ALT}(\mathrm{U} / \mathrm{L})$ & $62.54 \pm 10.96$ & $26.73 \pm 2.06$ & 0.008 \\
\hline TChol (mg/dL) & $207.92 \pm 6.92$ & $182.70 \pm 8.39$ & 0.023 \\
\hline HDL-Chol (mg/dL) & $59.89 \pm 3.20$ & $39.66 \pm 1.76$ & 0.000 \\
\hline LDL-Chol (mg/dL) & $118.32 \pm 5.67$ & $110.70 \pm 6.45$ & 0.381 \\
\hline TG (mg/dL) & $185.61 \pm 17.9$ & $167.17 \pm 12.54$ & 0.439 \\
\hline Glucose (mg/dL) & $86.76 \pm 1.47$ & $87.80 \pm 1.64$ & 0.641 \\
\hline ESR (mm/hour) & $13.31 \pm 0.93$ & $5.80 \pm 1.06$ & 0.000 \\
\hline CRP (mg/dL) & $0.89 \pm 0.08$ & $0.18 \pm 0.04$ & 0.000 \\
\hline Hemoglobin, g/L & $14.78 \pm 0.22$ & $15.33 \pm 0.15$ & 0.067 \\
\hline WBC count & $8585.71 \pm 449$ & $7668 \pm 342$ & 0.135 \\
\hline RDW & $13.91 \pm 0.19$ & $13.67 \pm 0.16$ & 0.369 \\
\hline MPV & $8.58 \pm 0.19$ & $7.71 \pm 0.23$ & 0.004 \\
\hline Neutrophil count & $5305.52 \pm 355$ & $4386.33 \pm 242$ & 0.054 \\
\hline Lymphocyte count & $2221.95 \pm 115$ & $2386.33 \pm 136$ & 0.361 \\
\hline Creatinine, $\mathrm{mg} / \mathrm{dl}$ & $0.79 \pm 0.01$ & $0.86 \pm 0.02$ & 0.008 \\
\hline Duration of heavy drinking year (years) & $20.38 \pm 1.37$ & 0 & - \\
\hline Daily ethanol consumption (g) & $186.64 \pm 7.0$ & 0 & - \\
\hline TLDE (kg ethanol/kg body weight) & $19.94 \pm 12.17$ & 0 & - \\
\hline
\end{tabular}

BMI: body mass index, CRP: C-reactive protein, ESR: erythrocyte sedimentation rate, WBC: white blood cell, RDW: red cell distribution width, MPV: mean platelet volume, AST: aspartic acid transaminase, ALT: alanine transaminase, TLDE: total lifetime dose of ethanol.

Data are expressed as mean \pm standard deviation.

include myocyte death, intracellular dysfunction, deterioration of mitochondrial ultrastructure, and sarcoplasmic reticulum [5]. Also it has been shown that cardiac myofibril shortening, composition of contractile proteins, and calcium homeostasis were adversely affected by excessive long term alcohol consumption [6]. These myocardial changes could lead to alcoholic cardiomyopathy later. In previous studies alcoholic patients with alteration in myocardial structure and function had a history of consuming $>90 \mathrm{~g} / \mathrm{d}$ of alcohol for 15 years [7-11]. It has been suggested that duration of daily alcohol consumption is more important than amount of daily alcohol consumption in developing heart failure [12, 13]. In our present study, alcoholic patients have consumed a mean daily dose of ethanol $186.64 \pm 7 \mathrm{~g}$ over a period of $20.38 \pm 1.37$ years.

The subclinical cardiac damage in chronic alcoholic patients was investigated in various studies using conventional and tissue Doppler echocardiography. Previous clinical studies evaluating the effect of chronic alcohol consumption on LV systolic and diastolic functions showed conflicting results. Impaired ejection fraction, preserved ejection fraction, and normal and impaired LV filling parameters were reported as a result in these studies [14-19].
Although the natural course of chronic alcoholic cardiomyopathy could not be defined clearly, it has been shown that abstaining from alcohol is associated with improvement in ejection fraction [20, 21]. As most of the alcoholics remain asymptomatic in the early stage of disease progression, early detection of subclinical LV dysfunction may lead to identifying patients at higher risk for heart failure and lead to interventions which would improve the health of alcoholic patients in entire society.

Two-dimensional speckle tracking echocardiography (STE) is a new noninvasive ultrasound imaging technique which allows measuring LV rotation, torsion, and strain as an evaluation of global and regional myocardial function. Speckle tracking echocardiography is angle independent and is less affected by artifacts, acoustic noise, and translational movements $[22,23]$. Speckle tracking echocardiographic parameters have been shown to detect subclinical regional and global myocardial dysfunction at an early stage in contrast to evaluation of ejection fraction by conventional methods [24, 25].

In our study all asymptomatic alcoholics had normal cardiac structure and ejection fraction, except that alcoholic patients had a significant difference in left atrial diameter. 
TABLE 2: Conventional echocardiographic data of alcoholic patients and controls.

\begin{tabular}{|c|c|c|c|}
\hline & $\begin{array}{c}\text { Control group } \\
n=30\end{array}$ & $\begin{array}{c}\text { Alcoholic group } \\
n=41\end{array}$ & $p$ \\
\hline $\mathrm{ARD}(\mathrm{mm})$ & $29.67 \pm 1.40$ & $30.33 \pm 1.40$ & 0.252 \\
\hline LV-EDD (mm) & $46.80 \pm 1.77$ & $48.21 \pm 1.61$ & 0.150 \\
\hline LV-ESD $(\mathrm{mm})$ & $28.33 \pm 1.71$ & $29.17 \pm 1.57$ & 0.359 \\
\hline $\mathrm{EF} \%$ & $70.33 \pm 1.06$ & $69.74 \pm 1.03$ & 0.695 \\
\hline FS\% & $39.93 \pm 2.87$ & $39.71 \pm 2.86$ & 0.862 \\
\hline IVS $(\mathrm{cm})$ & $0.95 \pm 0.16$ & $0.96 \pm 0.15$ & 0.457 \\
\hline PWT (cm) & $0.94 \pm 0.20$ & $0.90 \pm 0.20$ & 0.219 \\
\hline $\mathrm{LAD}(\mathrm{mm})$ & $31.83 \pm 0.47$ & $34.13 \pm 0.56$ & 0.003 \\
\hline Mit E wave velocity (m/s) & $0.77 \pm 0.16$ & $0.78 \pm 0.16$ & 0.775 \\
\hline Mit E DT (ms) & $204.01 \pm 6.17$ & $186.14 \pm 4.49$ & 0.019 \\
\hline Mit A wave velocity (m/s) & $0.71 \pm 0.16$ & $0.64 \pm 0.12$ & 0.03 \\
\hline $\mathrm{E} / \mathrm{A}$ & $1.14 \pm 0.37$ & $1.28 \pm 0.39$ & 0.120 \\
\hline $\mathrm{ET}(\mathrm{msn})$ & $250.20 \pm 28.84$ & $253.76 \pm 24.01$ & 0.439 \\
\hline IVRT (msn) & $78.97 \pm 21.04$ & $76.98 \pm 20.58$ & 0.393 \\
\hline IVCT (msn) & $57.17 \pm 15.15$ & $56.62 \pm 22.39$ & 0.772 \\
\hline MPI & $0.55 \pm 0.15$ & $0.53 \pm 0.18$ & 0.210 \\
\hline
\end{tabular}

ARD: aortic root diameter, LV: left ventricular, EDD: end diastolic diameter, ESD: end systolic diameter, EF: ejection fraction, FS: fractional shortening, IVS: septal thickness at end diastole, PWT: posterior wall thickness at end diastole, LAD: left atrium diameter, DT: deceleration time, ET: ejection time, IVRT: isovolumetric relaxation time, IVCT: isovolumetric contraction time, MPI: Myocardial Performance Index.

TABLE 3: Left ventricular strain measurements of alcoholic patients and controls.

\begin{tabular}{lccc}
\hline & Control group & Alcoholic group & $n=41$ \\
& $n=30$ & $-17.97 \pm-6.38$ & 0.001 \\
LV global longitudinal strain (\%) & $-19.97 \pm-7.37$ & $-17.17 \pm-4.64$ & 0.000 \\
LV global circumferential strain (\%) & $-21.18 \pm-3.78$ & $45.03 \pm 7.63$ & 0.596 \\
LV global radial strain (\%) & $46.76 \pm 6.94$ & & \\
\hline
\end{tabular}

Similar to our study, Singh et al. demonstrated that modest levels of alcohol consumption were associated with significant left atrium enlargement [26]. Different effects of alcohol consumption on lipoproteins in human and animal studies have been reported before. In previous studies, it has been shown that alcohol consumption is associated with increased levels of both HDL-2 and HDL-3 [27, 28]. Although we could not measure HDL subfractions in our current study, we found that HDL cholesterol levels were significantly higher in alcoholics than healthy controls. Experimental studies showed conflicting results about effects of alcohol on the inflammatory process. In epidemiologic studies it has been demonstrated that moderate alcohol consumption is related with low C-reactive protein (CRP) levels whereas high alcohol intake is associated with increased CRP levels. In the present study, increased CRP levels were observed in alcoholic patients group similar to previous studies [29, 30].

Our present study showed the limitations of conventional echocardiographic evaluation of early deterioration in cardiac systolic function and the value of speckle tracking echocardiography in subclinic alcoholic cardiomyopathy. The novel echocardiographic modalities would allow for demonstration of heavy alcohol consumption outcomes on systolic function.

The gross limitation of our study was the small sample size. Only male subjects were included in our study; therefore the effects of chronic alcoholism on LV functions in women could not be evaluated. Since there are no clear data related to classification, we could not group patients according to amount and duration of alcohol consumption for subgroup analysis. Clinical follow-up was not planned so the effect of subclinical dysfunction on cardiovascular prognosis remains unclear. The importance of these subclinical findings should be determined with a well-designed prospective study including cardiovascular prognosis.

\section{Conclusions}

Even with the abovementioned limitations we can conclude that speckle tracking echocardiography derived left ventricular systolic function is impaired in chronic alcoholic patients when compared with healthy controls. 


\section{Conflicts of Interest}

The authors declare that they have no conflicts of interest regarding the publication of this paper.

\section{References}

[1] A. George and V. M. Figueredo, "Alcoholic cardiomyopathy: a review," Journal of Cardiac Failure, vol. 17, no. 10, pp. 844-849, 2011.

[2] M. Dancy, G. Leech, J. M. Bland, M. K. Gaitonde, and J. D. Maxwell, "Preclinical left ventricular abnormalities in alcoholics are independent of nutritional status, cirrhosis, and cigarette smoking," The Lancet, vol. 325, no. 8438, pp. 1122-1125, 1985.

[3] A. Ammendola, M. R. Tata, C. Aurilio et al., "Peripheral neuropathy in chronic alcoholism: a retrospective cross-sectional study in 76 subjects," Alcohol and Alcoholism, vol. 36, no. 3, pp. 271-275, 2001.

[4] J. Brown, C. Jenkins, and T. H. Marwick, "Use of myocardial strain to assess global left ventricular function: a comparison with cardiac magnetic resonance and 3-dimensional echocardiography," American Heart Journal, vol. 157, no. 1, pp. 102.el102.e5, 2009.

[5] H. Klein and D. Harmjanz, "Effect of ethanol infusion on the ultrastructure of human myocardium," Postgraduate Medical Journal, vol. 51, no. 595, pp. 325-329, 1975.

[6] I. Laonigro, M. Correale, M. Di Biase, and E. Altomare, "Alcohol abuse and heart failure," European Journal of Heart Failure, vol. 11, no. 5, pp. 453-462, 2009.

[7] L. Fauchier, D. Babuty, P. Poret et al., "Comparison of long-term outcome of alcoholic and idiopathic dilated cardiomyopathy," European Heart Journal, vol. 21, no. 4, pp. 306-314, 2000.

[8] C. J. McKenna, M. B. Codd, H. A. McCann, and D. D. Sugrue, "Alcohol consumption and idiopathic dilated cardiomyopathy: a case control study," American Heart Journal, vol. 135, no. 5 I, pp. 833-837, 1998.

[9] A. M. Lazarević, S. Nakatani, A. N. Nešković et al., "Early changes in left ventricular function in chronic asymptomatic alcoholics: relation to the duration of heavy drinking," Journal of the American College of Cardiology, vol. 35, no. 6, pp. 1599$1606,2000$.

[10] M. Kupari, P. Koskinen, A. Suokas, and M. Ventilä, "Left ventricular filling impairment in asymptomatic chronic alcoholics," The American Journal of Cardiology, vol. 66, no. 20, pp. 14731477, 1990.

[11] M. R. Piano, "Alcoholic cardiomyopathy: incidence, clinical characteristics, and pathophysiology," Chest, vol. 121, no. 5, pp. 1638-1650, 2002.

[12] E. C. Mathews Jr., J. M. Gardin, W. L. Henry et al., "Echocardiographic abnormalities in chronic alcoholics with and without overt congestive heart failure," The American Journal of Cardiology, vol. 47, no. 3, pp. 570-578, 1981.

[13] A. Urbano-Marquez, R. Estruch, F. Navarro-Lopez, J. M. Grau, L. Mont, and E. Rubin, "The effects of alcoholism on skeletal and cardiac muscle," The New England Journal of Medicine, vol. 320, no. 7, pp. 409-415, 1989.

[14] P. Prazak, M. Pfisterer, S. Osswald, P. Buser, and F. Burkart, "Differences of disease progression in congestive heart failure due to alcoholic as compared to idiopathic dilated cardiomyopathy," European Heart Journal, vol. 17, no. 2, pp. 251-257, 1996.
[15] S. Mahmoud, L. M. Beauchesne, D. R. Davis, and C. Glover, "Acute reversible left ventricular dysfunction secondary to alcohol," Canadian Journal of Cardiology, vol. 23, pp. 475-477, 2007.

[16] K. Silberbauer, M. Juhasz, G. Ohrenberger, and C. Hess, "Noninvasive assessment of left ventricular diastolic function by pulsed doppler echocardiography in young alcoholics," Cardiology, vol. 75, no. 6, pp. 431-439, 1988.

[17] H. S. Friedman, B. C. Vasavada, A. M. Malec, K. K. Hassan, A. Shah, and S. Siddiqui, "Cardiac function in alcohol-associated systemic hypertension," The American Journal of Cardiology, vol. 57, no. 4, pp. 227-231, 1986.

[18] M. D. Cerqueira, G. D. Harp, J. L. Ritchie, J. R. Stratton, and R. D. Walker, "Rarity of preclinical alcoholic cardiomyopathy in chronic alcoholics < 40 years of age," The American Journal of Cardiology, vol. 67, no. 2, pp. 183-187, 1991.

[19] A. Askanas, M. Udoshi, and S. A. Sadjadi, "The heart in chronic alcoholism: a noninvasive study," American Heart Journal, vol. 99, no. 1, pp. 9-16, 1980.

[20] J. G. Demakis, A. Proskey, S. H. Rahimtoola et al., "The natural course of alcoholic cardiomyopathy," Annals of Internal Medicine, vol. 80, no. 3, pp. 293-297, 1974.

[21] P. Guillo, J. Mansourati, B. Maheu et al., "Long-term prognosis in patients with alcoholic cardiomyopathy and severe heart failure after total abstinence," American Journal of Cardiology, vol. 79, no. 9, pp. 1276-1278, 1997.

[22] G. Perk, P. A. Tunick, and I. Kronzon, "Non-doppler twodimensional strain imaging by echocardiography-from technical considerations to clinical applications," Journal of the American Society of Echocardiography, vol. 20, no. 3, pp. 234243, 2007.

[23] H. Geyer, G. Caracciolo, H. Abe et al., "Assessment of myocardial mechanics using speckle tracking echocardiography: fundamentals and clinical applications," Journal of the American Society of Echocardiography, vol. 23, no. 4, pp. 351-369, 2010.

[24] G. Roul, C. Cohen, and A. Lieber, "Anthracycline-induced cardiomyopathy," La Presse Médicale, vol. 38, no. 6, pp. 987-994, 2009 (French).

[25] J. L. Hare, J. K. Brown, R. Leano, C. Jenkins, N. Woodward, and T. H. Marwick, "Use of myocardial deformation imaging to detect preclinical myocardial dysfunction before conventional measures in patients undergoing breast cancer treatment with trastuzumab," The American Heart Journal, vol. 158, no. 2, pp. 294-301, 2009.

[26] K. J. Singh, B. E. Cohen, B. Na, M. Regan, N. B. Schiller, and M. A. Whooley, "Alcohol consumption and 5-year change in left atrial volume among patients with coronary heart disease: results from the heart and soul study," Journal of Cardiac Failure, vol. 19, no. 3, pp. 183-189, 2013.

[27] J. M. Gaziano, J. E. Buring, J. L. Breslow et al., "Moderate alcohol intake, increased levels of high-density lipoprotein and its subfractions, and decreased risk of myocardial infarction," New England Journal of Medicine, vol. 329, no. 25, pp. 1829-1834, 1993.

[28] B. A. Clevidence, M. E. Reichman, J. T. Judd et al., "Effects of alcohol consumption on lipoproteins of premenopausal women. A controlled diet study," Arteriosclerosis, Thrombosis, and Vascular Biology, vol. 15, no. 2, pp. 179-184, 1995.

[29] M. De Lorgeril, P. Salen, J.-L. Martin, F. Boucher, F. Paillard, and J. De Leiris, "Wine drinking and risks of cardiovascular complications after recent acute myocardial infarction," Circulation, vol. 106, no. 12, pp. 1465-1469, 2002. 
[30] A. Imhof, M. Froehlich, H. Brenner, H. Boeing, M. B. Pepys, and W. Koenig, "Effect of alcohol consumption on systemic markers of inflammation," The Lancet, vol. 357, no. 9258, pp. 763-767, 2001. 


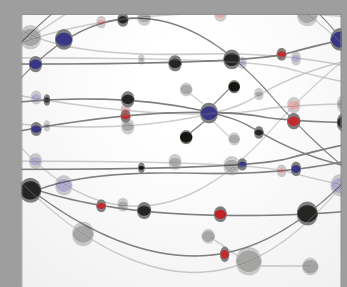

The Scientific World Journal
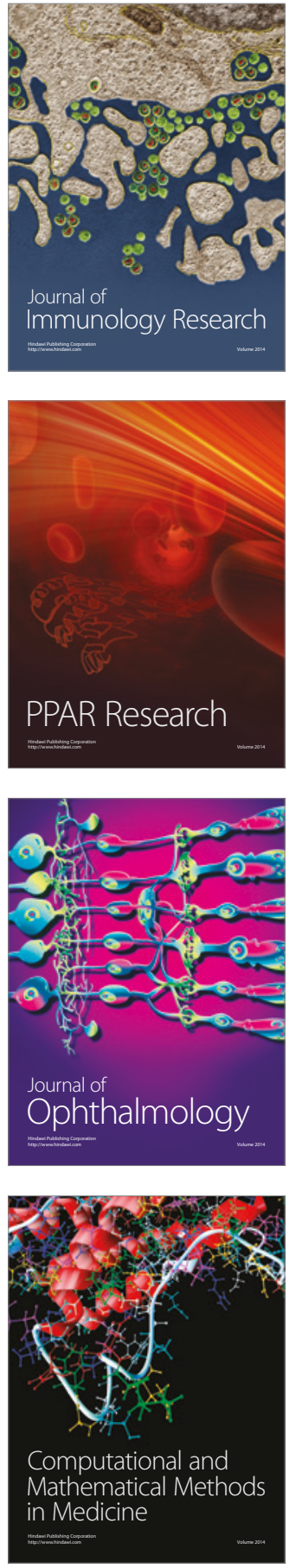

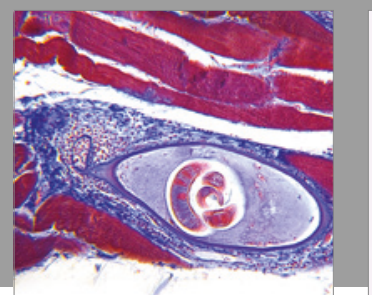

Gastroenterology Research and Practice
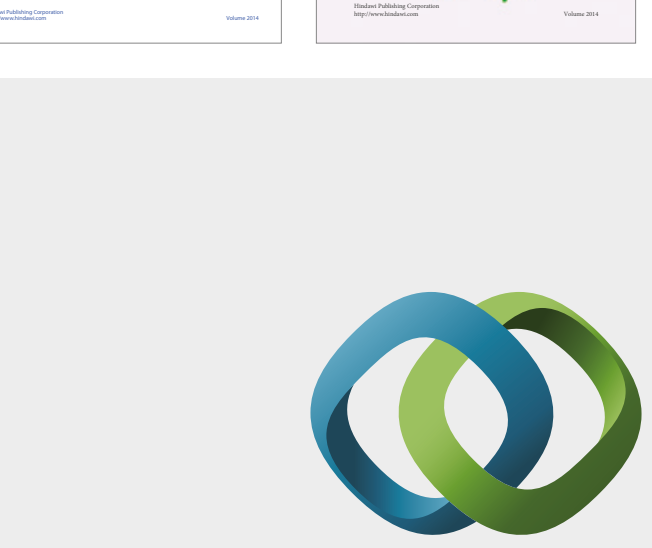

\section{Hindawi}

Submit your manuscripts at

https://www.hindawi.com
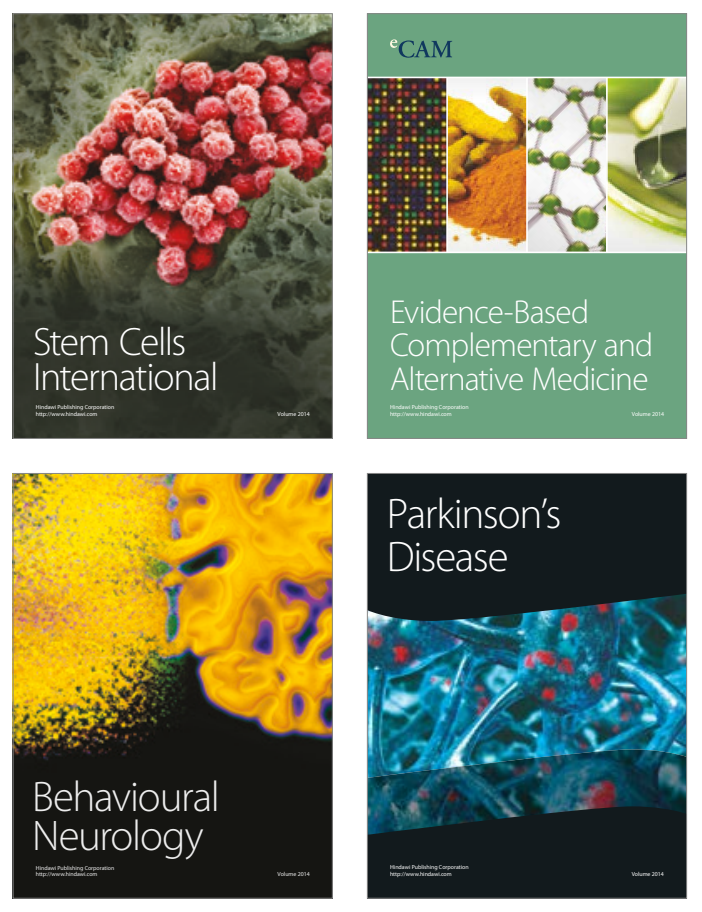
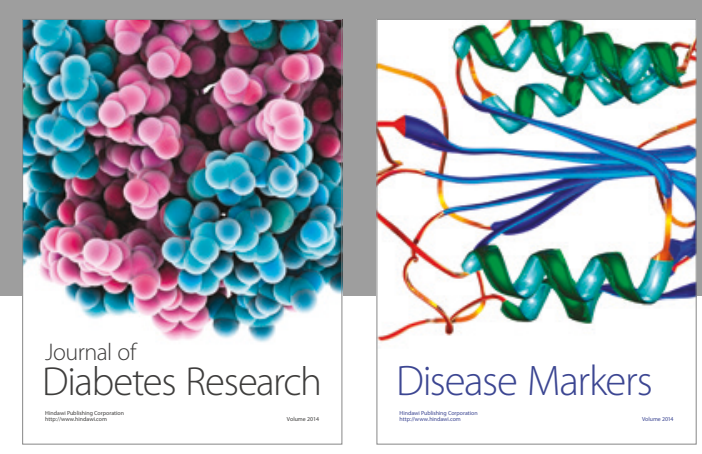

Disease Markers
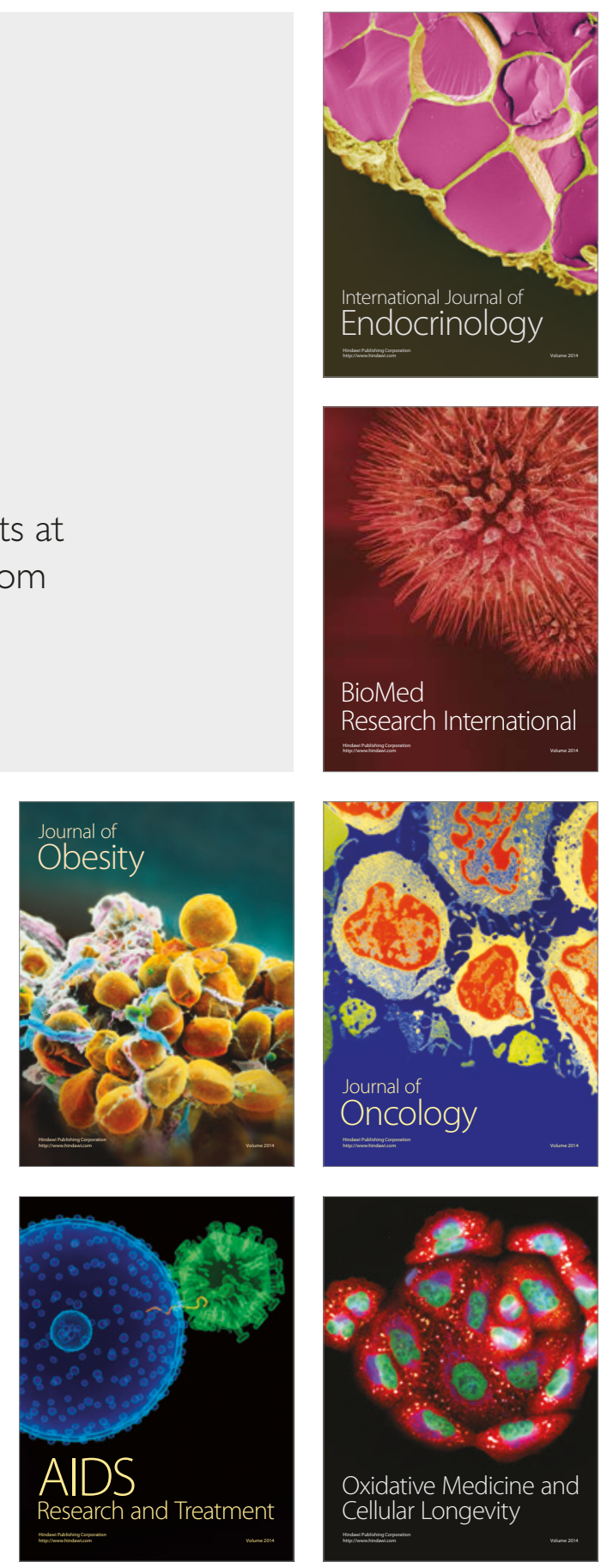